\title{
ATR prevents premature apoptosis
}

c9

the UV light-

induced

ATR-H cisconformation

has a direct anti-apoptotic role in mitochondria
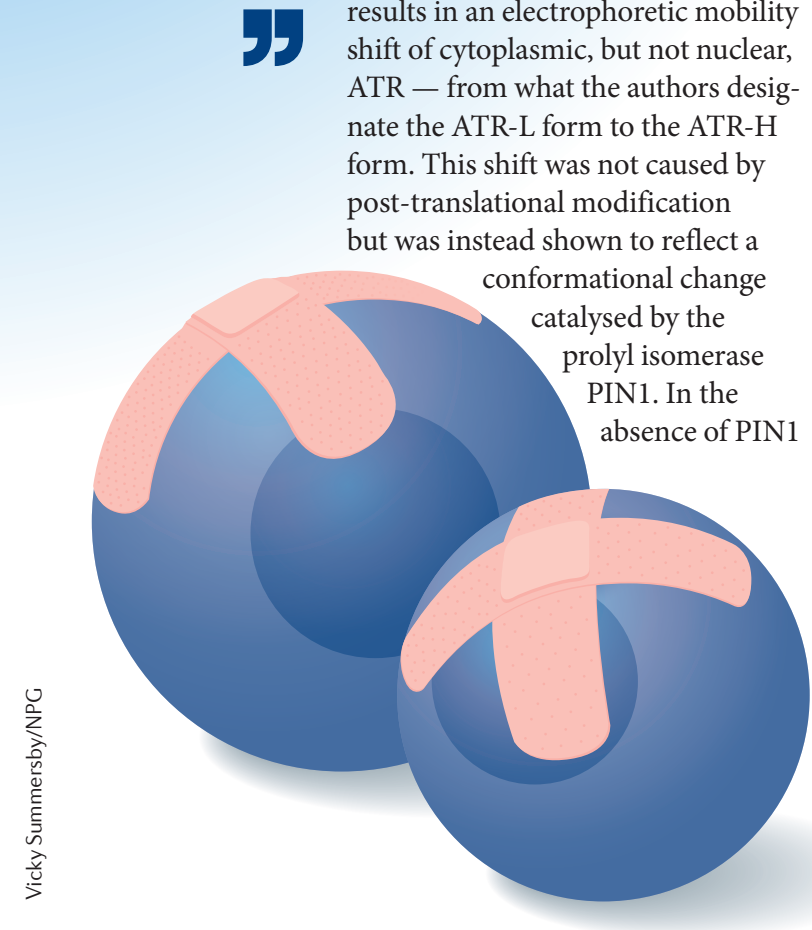

catalytic activity, cytoplasmic ATR was predominantly of the ATR-H cisconformation, even in the absence of UV irradiation. The results suggest that PIN1 maintains the ATR-L trans-conformation under normal conditions. This was confirmed in an in vitro isomerization assay, which further showed that phosphorylation of ATR-H is required for PIN1 binding, and that UV irradiation leads to the phosphorylation and inactivation of PIN1 and possibly to the dephosphorylation of ATR-H.

The combined effect of UV irradiation is therefore to prevent PIN1-mediated ATR-H to ATR-L isomerization. Following UV irradiation, the accumulated cytoplasmic ATR-H was localized exclusively to the mitochondrial outer membrane through direct interaction with the apoptotic protein BID. ATR contains three potential $\mathrm{BH} 3$-like domains, of which the second domain (amino acids 462-474) was required for ATR-H-BID interaction. The PIN1induced isomerization of ATR-H to ATR-L results in a conformational change of the amino-terminal region of ATR that is thought to sequester this $\mathrm{BH} 3$-like domain and prevent binding to BID.

The pro-apoptotic protein BAX is known to be activated by its binding to truncated BID (tBID) on mitochondria, resulting in cytochrome $c$ release and apoptosis. However, the accumulation of BID on mitochondria after UV irradiation was accompanied by BAX accumulation only in the absence of ATR, which suggests that BID binding by ATR-H might prevent BAX-tBID interactions. In line with this, ATR-H significantly decreased cytochrome $c$ release from isolated mitochondria incubated with tBID. The survival of UV-irradiated cells was decreased by mutating the $\mathrm{BH} 3$-like domain of ATR and was increased by PIN1 depletion. Of note, these mitochondrial effects of ATR are independent of its kinase activity and its nuclear co-factor ATRIP.

Together, the data indicate that the UV light-induced ATR-H cis-conformation has a direct antiapoptotic role in mitochondria by preventing the activation of BAX. This could prolong cell survival while (nuclear ATR-mediated) DNA repair is attempted. In healthy cells, PIN1 converts ATR-H to ATR-L, which allows for BAX-mediated apoptosis in response to appropriate signals.

Kirsty Minton

ORIGINAL RESEARCH PAPER Hilton, B. A. et al.

ATR plays a direct antiapoptotic role at

mitochondria, which is regulated by prolyl isomerase Pin1. Mol. Cell http://dx.doi. org/10.1016/j.molcel.2015.08.008 (2015) 